TRANSACTIONS OF THE

AMERICAN MATHEMATICAL SOCIETY

Volume 215, 1976

\title{
EQUIVARIANT BORDISM AND SMITH THEORY. IV
}

BY

R. E. STONG

ABSTRACT. This paper analyzes two types of characteristic numbers defined for manifolds with $Z_{\mathbf{4}}$ action, showing their relation and that neither suffices to detect $Z_{4}$ equivariant bordism. This extends work of Bix who had given examples not detected by one type of number.

1. Introduction. T. tom Dieck [4] has introduced a notion of equivariant Stiefel-Whitney numbers for actions of a finite group $G$ on closed manifolds and has shown that these numbers determine equivariant $G$ bordism for $G=$ $\left(Z_{2}\right)^{k}$. Recently, Bix [1] has shown that these numbers do not determine the bordism class for $G=Z_{4}$.

In [2], a notion of equivariant characteristic numbers for $Z_{2}$ actions was introduced. In this paper that notion will be extended, in the obvious way, to $G$ actions. It will then be shown that these numbers determine tom Dieck's numbers. For $Z_{4}$ they give more information than tom Dieck's numbers, but also do not detect bordism.

The author is indebted to Michael Bix for having made an advance copy of his paper available, and to the National Science Foundation for financial support.

2. Characteristic numbers. Let $G$ be a finite group; let $R, V_{1}, \ldots$, $V_{m}$ be the distinct irreducible real representations of $G$, with $R$ the trivial representation. Form $V=R^{\infty} \oplus V_{1}^{\infty} \oplus \cdots \oplus V_{m}^{\infty}$, the direct sum of a countable number of copies of each representation and let $B O_{n}$ be the Grassmannian of $n$-planes in $V$, with the $G$ action $\phi$ induced by the representation on $V$. Then $\left(B O_{n}, \phi\right)$ is a classifying space for $n$-plane bundles with $G$ action over decent spaces.

Letting $V \rightarrow R \oplus V: v \rightarrow(0, v)$ and identifying $R \oplus R^{\infty}$ with $R^{\infty}$ one assigns to an $n$-plane $\alpha$ in $V$ the $n+1$ plane $R \oplus \alpha$ in $R \oplus V \cong V$, which defines an inclusion $i: B O_{n} \rightarrow B O_{n+1}$. This is an equivariant map, and if $\gamma_{n}$ is the universal $n$-plane bundle over $B O_{n}, i^{*}\left(\gamma_{n+1}\right)=\gamma_{n} \oplus 1_{+}$, where $1_{+}$is the trivial line bundle with trivial $G$ action. Let $(B O, \phi)$ be the limit of the $B O_{n}$ 's with these maps.

If $(M, \psi)$ is a compact $n$ manifold with boundary with $G$ action, there is a

Received by the editors January 27, 1975.

AMS (MOS) subject classifications (1970). Primary 57D85. 
classifying map $\tau_{M}:(M, \psi) \rightarrow\left(B O_{n}, \phi\right)$, unique up to equivariant homotopy. If $(M, \psi)$ is a regularly imbedded invariant submanifold of $\partial V$, with $\left(V, \psi^{\prime}\right)$ a $G$ action, then the tangent bundle of $V$ restricts to $\tau_{M} \oplus 1_{+}$on $M$, which is classified by $i \circ \tau_{M}:(M, \psi) \rightarrow\left(B O_{n+1}, \phi\right)$. Thus one has a well-defined homotopy class of maps $\tau_{M}:(M, \psi) \rightarrow(B O, \phi)$ classifying the stable tangent bundle of $M$, and if $(M, Q)$ is regularly imbedded in the boundary of $\left(V, \psi^{\prime}\right)$ by $f: M \rightarrow \partial V$, then $\tau_{V} \circ f=\tau_{M}$.

Being given a pair $(X, A, \rho)$ with $G$ action one then defines a natural transformation $\tau_{*}: \Re_{*}^{G}(X, A, \rho) \rightarrow \Re_{*}^{G}(X \times B O, A \times B O, \rho \times \phi)$ by sending the class of $f:(M, \partial M, \psi) \rightarrow(X, A, \rho)$ to the class of $f \times \tau_{M}:(M, \partial M, \psi) \rightarrow(X \times B O$, $A \times B O, \rho \times \phi)$.

For any pair $(X, A, \rho)$, one has a natural transformation

$$
\mu: \Re_{*}^{G}(X, A, \rho) \rightarrow H_{*}^{G}\left(X, A, \rho ; Z_{2}\right)
$$

assigning to $f:(M, \partial M, \psi) \rightarrow(X, A, \rho)$ the image $f_{*}([M, \partial M, \psi])$ of the fundamental Smith homology class (see $[3, \S 2])$.

The composite

$$
\mu^{\circ} \tau_{*}: \mathfrak{N}_{*}^{G}(X, A, \rho) \rightarrow H_{*}^{G}\left(X \times B O, A \times B O, \rho \times \phi ; Z_{2}\right)
$$

gives rise to equivariant characteristic numbers; i.e. every element of the dual Smith cohomology group $H_{G}^{*}\left(X \times B O, A \times B O, \rho \times \phi ; Z_{2}\right)$ gives a characteristic number for the $G$ bordism of $(X, A, \rho)$. Restricting to $X$ a point and $A$ empty gives $\mu \circ \tau_{*}: \mathfrak{N}_{*}^{G} \rightarrow H_{*}^{G}\left(B O, \phi ; Z_{2}\right)$.

3. Tom Dieck's numbers. To describe tom Dieck's characteristic numbers, one may follow Bix's approach. Let $\pi: E G \rightarrow B G$ be a universal principal $G$ bundle and let $(M, \psi)$ be a $G$ manifold with $\left(\tau(M), \psi_{*}\right)$ its tangent bundle with $G$ action induced by the differential. Then $E G \times_{G} \tau(M) \rightarrow E G \times_{G} M$ is a vector bundle classified by a map $E G \times_{G} M \stackrel{\alpha}{\longrightarrow} B O$, inducing a homomorphism

$$
\alpha^{*}: H^{*}\left(B O ; Z_{2}\right) \rightarrow H^{*}\left(E G \times_{G} M ; Z_{2}\right) \text {. }
$$

Integration along the fibers defines a homomorphism

$$
\text { ६: } H^{*}\left(E G \times_{G} M ; Z_{2}\right) \rightarrow H^{*}\left(B G ; Z_{2}\right)
$$

of degree $-(\operatorname{dim} M)$. Assigning to $(M, \psi)$ the homomorphism :० $\alpha^{*}$ defines a homomorphism

$$
\chi: \Re_{*}^{G} \rightarrow \operatorname{Hom}\left(H^{*}\left(B O ; Z_{2}\right), H^{*}\left(B G ; Z_{2}\right)\right)
$$

which gives characteristic numbers.

To relate this to Smith homology, consider a space with $G$ action $(X, \phi)$. Let $C(X)$ denote the mod 2 chains of $X$ and $C^{\circ}(X)$ the subcomplex of chains 
invariant under $G$. Then $H_{*}^{G}\left(X ; Z_{2}\right)$ is the homology of the complex $C^{\circ}(X)$. In particular, if $G$ acts freely on $X, C^{\circ}(X)$ is isomorphic to $C(X / G)$ and $H_{*}^{G}\left(X ; Z_{2}\right)$ $\cong H_{*}\left(X / G ; Z_{2}\right)$.

Now consider a class $a \in H_{*}^{G}\left(X ; Z_{2}\right)$ and a class $b \in H_{*}\left(B G ; Z_{2}\right)$. Representative cycles $a^{\prime}$ and $b^{\prime}$ may be chosen in $C^{\circ}(X)$ and $C^{\circ}(E G)$, and their product $a^{\prime} \otimes b^{\prime}$ is a cycle in $C^{\circ}(X \times E G)$, giving a class in $H_{*}\left(X \times{ }_{G} E G ; Z_{2}\right)$. Thus one has a product

$$
H_{*}^{G}\left(X ; Z_{2}\right) \otimes H_{*}\left(B G ; Z_{2}\right) \rightarrow H_{*}\left(X \times_{G} E G ; Z_{2}\right)
$$

or a homomorphism

$$
H_{*}^{G}\left(X ; Z_{2}\right) \rightarrow \operatorname{Hom}\left(H_{*}\left(B G ; Z_{2}\right), H_{*}\left(X \times_{G} E G ; Z_{2}\right)\right)
$$

and applying duality of homology and cohomology gives

$$
\cap: H_{*}^{G}\left(X ; Z_{2}\right) \rightarrow \operatorname{Hom}\left(H^{*}\left(X \times_{G} E G ; Z_{2}\right), H^{*}\left(B G ; Z_{2}\right)\right) \text {. }
$$

Notice that if $X$ is given by a $G$ manifold $(M, \psi)$, that $\bigcap([M])$ is just 4 , i.e. integration along the fibers is obtained in this way from the fundamental class of $M$.

Now letting $(B O, \phi)$ be the universal action with universal $G$ bundle $\left(\gamma, \phi_{*}\right)$, $E G \times{ }_{G} \gamma \rightarrow E G \times{ }_{G} B O$ is a vector bundle, classified by a map $c: E G \times{ }_{G} B O$ $\rightarrow B O$. Assigning to $x \in H_{*}^{G}\left(B O, \phi ; Z_{2}\right)$ the composite $\bigcap(x) \circ c^{*}$ defines a homomorphism

$$
\gamma^{*}: H_{*}^{G}\left(B O, \phi ; Z_{2}\right) \rightarrow \operatorname{Hom}\left(H^{*}\left(B O ; Z_{2}\right), H^{*}\left(B G ; Z_{2}\right)\right) .
$$

Proposition 3.1. The homomorphism $\chi$ is the composite of

and

$$
\mu \circ \tau_{*}: \mathfrak{N}_{*}^{G} \rightarrow H_{*}^{G}\left(B O, \phi ; Z_{2}\right)
$$

$$
\gamma^{*}: H_{*}^{G}\left(B O, \phi ; Z_{2}\right) \rightarrow \operatorname{Hom}\left(H^{*}\left(B O ; Z_{2}\right), H^{*}\left(B G ; Z_{2}\right)\right)
$$

Proof. Given $(M, \psi)$ with $\tau:(M, \psi) \rightarrow(B O, \phi)$ classifying the tangent bundle, the composite

$$
E G \times{ }_{G} M \stackrel{1 \times \tau}{\longrightarrow} E G \times{ }_{G} B O \stackrel{c}{\longrightarrow} B O
$$

is the map $\alpha$. Then $\circ \circ \alpha^{*}=\bigcap([M]) \circ \alpha^{*}=\bigcap([M]) \circ(1 \times \tau)^{*} \circ c^{*}=$ $\bigcap\left(\tau_{*}[M]\right) \circ c^{*}=\bigcap\left(\mu \circ \tau_{*}(M, \psi)\right) \circ c^{*}=\gamma^{*}\left(\mu \circ \tau_{*}(M, \psi)\right)$, using the obvious naturality property of $\cap . *$

4. Actions of $Z_{4}$. First one needs to know the structure of $H_{*}^{Z_{4}}\left(B O, \phi ; Z_{2}\right)$. As noted in [3, Lemma 2.1], if $T \subset G$ is a central subgroup of order 2, then 


$$
H_{*}^{G}\left(X, A, \rho ; Z_{2}\right) \cong H_{*}^{G}\left(X, F_{T} \cup A, \rho ; Z_{2}\right) \oplus H_{*}^{G}\left(F_{T}, F_{T} \cap A, \rho ; Z_{2}\right)
$$

where $F_{T}$ is the fixed set of $T$, and

$$
\begin{gathered}
H_{*}^{G}\left(X, F_{T} \cup A, \rho ; Z_{2}\right) \cong H_{*}^{G / T}\left(X / T,\left(F_{T} \cup A\right) / T, \rho^{\prime} ; Z_{2}\right) \\
H_{*}^{G}\left(F_{T}, F_{T} \cap A, \rho ; Z_{2}\right) \cong H_{*}^{G / T}\left(F_{T}, F_{T} \cap A, \rho^{\prime} ; Z_{2}\right)
\end{gathered}
$$

where $\rho^{\prime}$ is induced by $\rho$. In particular, for $G=Z_{4}$, one may take $T=Z_{2}$, and one needs to know about the fixed set of $Z_{2}$ on $B O$.

Now the irreducible representations of $Z_{4}$ are $R, R_{-}$, and $C$, where $R_{-}$is the reals with $Z_{4}$ acting as multiplication by -1 and $C$ is the complex numbers with $Z_{4}$ acting as multiplication by $i$. The fixed set of $Z_{2}$ on $B O_{n}$ is then $\bigcup_{k} B O_{n-k}\left(R^{\infty} \oplus R_{-}^{\infty}\right) \times B O_{k}\left(C^{\infty}\right)$ and taking the limit, the fixed set $F$ of $Z_{2}$ on $B O$ is $\bigcup B O\left(R^{\infty} \oplus R_{-}^{\infty}\right) \times B O_{k}\left(C^{\infty}\right)$. The induced $Z_{2}$ action on $F$ preserves these components and in particular, $B O\left(R^{\infty} \oplus R_{-}^{\infty}\right) \times B O_{0}\left(C^{\infty}\right)=B O$ is the universal space for $Z_{2}$ bundles.

The pair $(B O, F)$ is relatively a free action, hence may be crossed with $E Z_{4}$ giving an isomorphism

$$
\begin{aligned}
H_{*}^{Z_{4}}\left(B O, F, \phi ; Z_{2}\right) & \cong H_{*}^{Z_{4}}\left(B O \times E Z_{4}, F \times E Z_{4}, \phi \times u ; Z_{2}\right) \\
& \cong H_{*}^{Z}\left(\left(B O \times E Z_{4}\right) / Z_{2}, F \times\left(E Z_{4} / Z_{2}\right),(\phi \times u)^{\prime} ; Z_{2}\right) \\
& \cong H_{*}\left(\left(B O \times E Z_{4}\right) / Z_{4},\left(F \times E Z_{4} / Z_{2}\right) / Z_{2} ; Z_{2}\right) .
\end{aligned}
$$

Now $B O \times E Z_{4} / Z_{4}$ maps into $B Z_{4}=E Z_{4} / Z_{4}$ by projection with fiber $B O$, and maps by $c$ (of $\S 3$ ) into $B O$. Looking at the fixed component $B O \times B O_{0}$ of $F$, the map $\left(B O \times B O_{0} \times\left(E Z_{4} / Z_{2}\right)\right) / Z_{2} \rightarrow B O \times E Z_{4} / Z_{4}$ is a homotopy equivalence, both being compatibly homotopy equivalent to $B O \times B Z_{4}$. Thus $B O \times B O_{0} \times$ $E Z_{4} \rightarrow B O \times E Z_{4}$ is an equivariant homotopy equivalence. Thus the exact sequence for the pair $\left(B O \times E Z_{4}, F \times E Z_{4}\right)$ decomposes, and the boundary homomorphism

ว: $H_{*}^{Z_{4}}\left(B O, F, \phi ; Z_{2}\right)$

$$
\rightarrow \bigoplus_{k \neq 0} H_{*-1}^{Z_{2}}\left(B O\left(R^{\infty} \oplus R_{-}^{\infty}\right) \times B O_{k}\left(C^{\infty}\right) \times\left(E Z_{4} / Z_{2}\right), \phi^{\prime} \times u^{\prime} ; Z_{2}\right)
$$

is an isomorphism.

Now one analyzes $\mathfrak{N}_{*}^{Z_{4}}$. One has an exact sequence

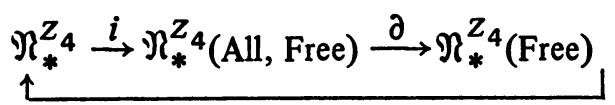

and a free action $(M, \psi)$ bounds the mapping cylinder of $M \rightarrow M / Z_{2}$. Now $\mathfrak{N}_{*}^{Z_{4}}$ (All, Free) may be computed by taking the fixed set of $Z_{2}$. The fixed set 
of $\left(M^{n}, \psi\right)$ is a union of closed submanifolds $F^{n-k}$ imbedded in the interior of $M$ with induced $Z_{2}=Z_{4} / Z_{2}$ action. The normal bundle of $F^{n-k}$ is a bundle with $Z_{4}$ action so that $Z_{2}$ acts as -1 in the fibers; i.e. $\nu$ is classified by an equivariant map $\nu: F^{n-k} \rightarrow B O_{k}\left(C^{\infty}\right)$. Thus

$$
\mathfrak{N}_{*}^{Z_{4}}(\mathrm{All}, \text { Free }) \cong \bigoplus_{k} \mathfrak{N}_{*-k}^{Z_{2}}\left(B O_{k}\left(C^{\infty}\right)\right) \text {. }
$$

The mapping cylinder splitting and $\partial$ give an isomorphism of $\mathfrak{N}_{*-1}^{Z_{4}}$ (Free) with the summand $\mathfrak{N}_{*-1}^{Z_{2}}\left(B O_{1}\left(C^{\infty}\right)\right)$.

From the splitting, one obtains an isomorphism

$$
P: \bigoplus_{k \neq 1} \mathfrak{N}_{*-k}^{Z_{2}}\left(B O_{k}\left(C^{\infty}\right)\right) \stackrel{\cong}{\cong} \mathfrak{N}_{*}^{Z_{4}}
$$

assigning to $\nu: F^{n-k} \rightarrow B O_{k}\left(C^{\infty}\right)$ the induced $Z_{4}$ action on $D(v) /(x \sim-x \mid x \in S(\nu))$, the real projective space bundle of $\nu \oplus 1$. On the summand $\mathfrak{N}_{*}^{Z_{2}}\left(B O_{0}\left(C^{\infty}\right)\right)$ $\cong \mathfrak{N}_{*}^{Z_{2} P}$ assigns to the involution $\left(M^{n}, t\right)$ the induced $Z_{4}$ action $\left(M, \phi_{t}\right)$ with $Z_{2} \subset Z_{4}$ acting trivially $(D(\nu)=M$ and $S(\nu)$ is empty).

First considering $\left(M^{n}, t\right)$ in $\mathfrak{N}_{*}^{Z_{2}}\left(B O_{0}\left(C^{\infty}\right)\right) . P\left(M^{n}, t\right)=\left(M, \phi_{t}\right)$ has all simplices fixed by $Z_{2}$ and hence $\mu^{\circ} \tau_{*} \circ P\left(M^{n}, t\right)$ lies in the summand $H_{*}^{Z_{4}}\left(F_{t}, \phi ; Z_{2}\right)$ of $H_{*}^{Z_{4}}\left(B O, \phi ; Z_{2}\right)$. The classifying map $\tau_{M}:\left(M, \phi_{t}\right) \rightarrow(B O, \phi)$ maps into the fixed set of $Z_{2}$, and may be obtained by composing the classifying map for the $Z_{2}$ tangent bundle $\tau_{M}:\left(M^{n}, t\right) \rightarrow\left(B O, \phi^{\prime}\right)$ with the inclusion $i$ of $(B O$, $\left.\phi^{\prime}\right)$ as $B O\left(R^{\infty} \oplus R_{-}^{\infty}\right) \times B O_{0}\left(C^{\infty}\right)$ in $F$, and then with the inclusion $j$ of $F$ in $B O$. It is then immediate that the diagram

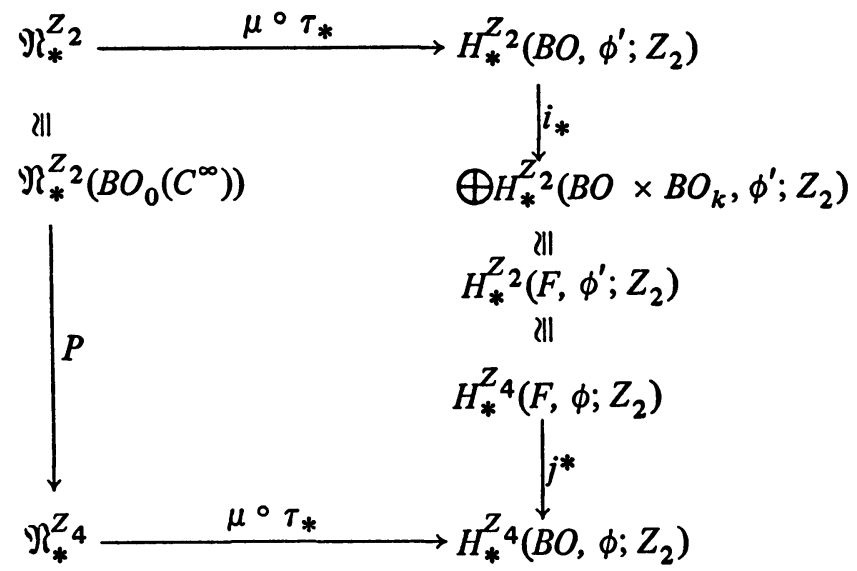

commutes. Since $\mu^{\circ} \tau_{*}$ is monic for $Z_{2}$ by [2, Proposition 3.1] and $i_{*}, j_{*}$ are monic, it follows that $\mu \circ \tau_{*} \circ P$ is monic on $\mathfrak{N}_{*}^{Z_{2}}\left(B O_{0}\left(C^{\infty}\right)\right)$.

For any class in the remaining summands, $\mathfrak{N}_{*-k}^{Z_{2}}\left(B O_{k}\left(C^{\infty}\right)\right), k \neq 0,1$, the action $P(\alpha)$ has no top dimensional simplices fixed under the action of $Z_{2}$. Thus $\mu \circ \tau_{*} \circ P$ sends $A=\bigoplus_{k \neq 0,1} \Re_{*-k}^{Z_{2}}\left(B O_{k}\left(C^{\infty}\right)\right)$ into the summand 
$H_{*}^{Z_{4}}\left(B O, F, \phi ; Z_{2}\right)$ of $H_{*}^{Z_{4}}\left(B O, \phi ; Z_{2}\right)$, which in turn maps isomorphically by $\partial$ to

$$
\bigoplus_{k \neq 0} H_{*-k}^{Z_{2}}\left(B O \times B O_{k}\left(C^{\infty}\right) \times E Z_{4} / Z_{2}, \phi^{\prime} \times u^{\prime} ; Z_{2}\right)
$$

Now considering $v: F^{n-k} \rightarrow B O_{k}\left(C^{\infty}\right)$, the fixed set of $Z_{2}$ in $D(\nu) /\left(x^{\sim}-x \mid x \in S(\nu)\right)$ consists of $F^{n-k}$ and $S(\nu) / Z_{2}=R P(\nu)$. The image of this class under $\partial \circ \mu^{\circ} \tau_{*} \circ P$ is the sum of the classes:

$\left(a^{\prime}\right)$ the image of the fundamental class of $R P(\nu)$ in

$$
H_{n-1}^{Z_{2}}\left(B O \times B O_{k}\left(C^{\infty}\right) \times\left(E Z_{4} / Z_{2}\right) ; \phi^{\prime} \times \mu^{\prime} ; Z_{2}\right)
$$

obtained by

$$
\tau \circ \pi \times \nu \circ \pi \times c: R P(\nu) \rightarrow B O \times B O_{k} \times\left(E Z_{4}\right) / Z_{2}
$$

classifying the pull back of the tangent bundle, the pull back of $\nu$, and the double cover of $R P(\nu)$ by $S(\nu)$.

(b') The image of the fundamental class of $R P(\lambda)$ in

$$
H_{n-1}^{Z_{2}}\left(B O \times B O_{1}\left(C^{\infty}\right) \times\left(E Z_{4} / Z_{2}\right) ; \phi^{\prime} \times \mu^{\prime} ; Z_{2}\right)
$$

obtained by $\tau \circ \pi \times \nu \circ \pi \times c: R P(\lambda) \rightarrow B O \times B O_{1} \times\left(E Z_{4} / Z_{2}\right)$ where $\pi$ is projection on the fixed component $R P(\nu)$ with normal bundle $\lambda$.

To see this, $\tau: M^{n} \rightarrow B O$ sends a fixed component $F^{n-k}$ with normal bundle $\nu^{k}$ into $B O \times B O_{k}\left(C^{\infty}\right)$ classifying $\tau \oplus \nu$. On a tubular neighborhood $D(\nu), \tau$ is homotopic to $\tau / F \circ \pi$, i.e. $\tau$ is the pull back of $\tau \oplus \nu$ and similarly on the boundary $S(\nu)$. Sending $M-F$ into $E Z_{4}$ to classify $M-F \rightarrow(M-F) / Z_{4}$ and dividing out $Z_{2}$ gives $R P(\nu) \rightarrow B O \times B O_{k}\left(C^{\infty}\right) \times E Z_{4} / Z_{2}$ classifying $\tau^{\circ} \pi$, $\nu \circ \pi$, and the cover by $S(\nu)$.

Over $B O \times B O_{k}\left(C^{\infty}\right) \times E Z_{4} / Z_{2}$ one has a stable bundle $\gamma$ with involution, a $k$ plane bundle $\rho$ with $Z_{4}$ action covering the involution on the base and a double cover or line bundle $\lambda$ with similar $Z_{4}$ action. Then $\rho \otimes \lambda$ is a $k$ plane bundle with involution and

$$
\alpha: B O \times B O_{k}\left(C^{\infty}\right) \times E Z_{4} / Z_{2} \rightarrow B O \times B O_{k}\left(C^{\infty}\right) \times E Z_{4} / Z_{2}
$$

where $\pi_{1} \circ \alpha$ classifies $\gamma \oplus(\rho \otimes \lambda), \pi_{2}^{\circ} \alpha=\pi_{2}, \pi_{3}^{\circ} \alpha=\pi_{3}$ is an equivariant homotopy equivalance, with inverse obtained by subtracting $\rho \otimes \lambda$.

Applying $\alpha_{*}$ sends the class ( $\left.\mathrm{a}^{\prime}\right)$ into the class (a) the image of the fundamental class of $R P(\nu)$ in $H_{n-1}^{Z_{2}}\left(B O \times B O_{k}\left(C^{\infty}\right) \times\left(E Z_{4} / Z_{2}\right), \phi^{\prime} \times \mu^{\prime} ; Z_{2}\right)$ obtained by $\tau \times \nu \circ \pi \times c: R P(\nu) \rightarrow B O \times B O_{k} \times\left(E Z_{4} / Z_{2}\right)$ classifying the tangent bundle of $R P(\nu)$, the pull back of $\nu$ and the double cover of $R P(\nu)$ by $S(\nu)$.

On the fixed component $R P(\nu)$, the normal bundle $\lambda$ is a line bundle, so $\pi$ : $R P(\lambda) \rightarrow R P(\nu)$ is a diffeomorphism. The map into $B O_{1}\left(C^{\infty}\right)=E Z_{4} / Z_{2}$ classifying 
$\nu \circ \pi$ is the same as the map $c$ classifying the double cover. Tensoring this bundle with itself gives a trivial bundle with trivial action (if $e$ is a unit vector over $x, e \otimes e$ is independent of the choice of $e$ and is sent by the $Z_{4}$ action into the point $e^{\prime} \otimes e^{\prime}$ if $e$ goes to $\left.e^{\prime}\right)$. Identifying $R P(\lambda)$ with $R P(\nu)$ via $\pi, \tau \circ \pi=$ $\tau \cong \tau \oplus(\lambda \otimes \lambda)$, so applying $\alpha_{*}$ sends the class $\left(b^{\prime}\right)$ into the class

(b) the image of the fundamental class of $R P(\nu)$ in

$$
H_{n-1}^{Z_{2}}\left(B O \times B O_{1}\left(C^{\infty}\right) \times\left(E Z_{4} / Z_{2}\right) ; \phi^{\prime} \times \mu^{\prime} ; Z_{2}\right)
$$

obtained by $\tau \times \hat{\nu} \times c: R P(\nu) \rightarrow B O \times B O_{1} \times\left(E Z_{4} / Z_{2}\right)$ classifying $\tau$, the normal bundle of $R P(\nu)$ in $D(v) / Z_{2}$ on $S(v)$, and the double cover by $S(v)$. Note: The last two maps are the same.

Note: The class given by (b) may be obtained from that in (a) by applying the map

$$
\begin{aligned}
B O \times B O_{k} \times\left(E Z_{4} / Z_{2}\right) & \stackrel{\pi}{\longrightarrow} B O \times\left(E Z_{4} / Z_{2}\right) \\
& \stackrel{\iota \times \Delta}{\longrightarrow} B O \times\left(E Z_{4} / Z_{2}\right) \times\left(E Z_{4} / Z_{2}\right)
\end{aligned}
$$

Further, the class (a) may be obtained from the composite of

$$
Q: \mathfrak{N}_{*-k}^{Z_{2}}\left(B O_{k}\left(C^{\infty}\right)\right) \rightarrow \mathfrak{N}_{*-1}^{Z_{2}}\left(B O_{k}\left(C^{\infty}\right) \times\left(E Z_{4} / Z_{2}\right)\right)
$$

assigning to $v: F^{n-k} \rightarrow B O_{k}$ the map $\nu \circ \pi \times c: R P(\nu) \rightarrow B O_{k} \times\left(E Z_{4} / Z_{2}\right)$ and the monomorphism

$$
\mu \circ \tau_{*}: \Re_{*-1}^{Z_{2}}\left(B O_{k} \times\left(E Z_{4}\right) / Z_{2}\right) \rightarrow H_{*-1}^{Z_{2}}\left(B O \times B O_{k} \times E Z_{4} / Z_{2}, \phi^{\prime} \times \mu^{\prime} ; Z_{2}\right) .
$$

Thus, one concludes that $\mu^{\circ} \tau_{*}$ is monic for $\mathfrak{N}_{*}^{Z_{4}}$ if and only if

$$
Q: \mathfrak{N}_{*-k}^{Z_{2}}\left(B O_{k}\left(C^{\infty}\right)\right) \rightarrow \mathfrak{N}_{*-1}^{Z_{2}}\left(B O_{k}\left(C^{\infty}\right) \times\left(E Z_{4} / Z_{2}\right)\right)
$$

is monic for each $k>1$.

For $k$ odd, $Q$ is monic. To see this, note that $Z_{2}$ acts freely on $B O_{k}\left(C^{\infty}\right)$ for a fixed point is a $k$ dimensional subspace of $C^{\infty}$ invariant under $i$, i.e. a complex subspace. Thus $\mathfrak{N}_{*-k}^{Z_{2}}\left(B O_{k}\left(C^{\infty}\right)\right) \cong \mathcal{N}_{*-k}\left(B O_{k} / Z_{2}\right)$. Further $\left(B O_{k} \times\left(E Z_{4} / Z_{2}\right)\right) / Z_{2}$ maps into $B O_{k} / Z_{2}$ and $E Z_{4} / Z_{4}=B Z_{4}$. Given $M^{n} \stackrel{\nu^{\prime}}{\longrightarrow}$ $B O_{k} / Z_{2}$, one has a double cover $\tilde{M} \stackrel{\nu}{\longrightarrow} B O_{k}$ and over $\tilde{M}$ the projective space bundle $R P(v)$. Dividing out $Z_{2}$ on $R P(v)$ gives a bundle $E \stackrel{\pi}{\longrightarrow} M$ with fiber $R P(k-1)$ and the corresponding class in

$$
\mathfrak{N}_{*-1}\left(B O_{k} / Z_{2} \times B Z_{4}\right) \text { is given by } \nu^{\prime} \circ \pi \times f: E \rightarrow B O_{k} / Z_{2} \times B Z_{4}
$$

where $f$ classifies the cover $S(\nu) \rightarrow E$.

Now the $R P(k-1)$ bundle $E \stackrel{\pi}{\longrightarrow} M$ is not totally nonhomologous to zero, 
but the map into $B Z_{4}$ induces the nontrivial four fold cover of $R P(k-1)$, and since $k$ is odd, this implies that for $\alpha \in H^{k-1}\left(B Z_{4}, Z_{2}\right)$ the nonzero class, $\alpha$ pulls back to the nonzero class in $H^{k-1}\left(R P(k-1) ; Z_{2}\right)$.

Let $f_{i}: M_{i}^{n_{i}} \rightarrow B O_{k} / Z_{2}$ be bordism elements giving a base for $\bmod 2$ homology, with dual base $\beta^{i} \in H^{n_{i}}\left(B O_{k} / Z_{2} ; Z_{2}\right)$. Then

$$
Q^{\prime}: \mathfrak{N}_{*}\left(B O_{k} / Z_{2}\right) \rightarrow \mathfrak{N}_{*+k-1}\left(B O_{k} / Z_{2} \times B Z_{4}\right)
$$

sends $\left[M_{i}^{n_{i}}, f_{i}\right]$ into linearly independent classes detected by the $\beta^{i} \otimes \alpha$. Since $Q^{\prime}$ is an $\Re_{*}$ module homomorphism, $Q^{\prime}$ is monic. Since $Q^{\prime}$ factors through $Q, Q$ is monic.

However, for $k$ even, $Q$ is not monic. The easiest example is to consider $R P(4)$ with the trivial involution $T_{0}$ and with $T_{1}\left(\left[x_{0}, x_{1}, \ldots, x_{4}\right]\right)=\left[-x_{0}\right.$, $\left.-x_{1}, \ldots, x_{4}\right]$, with a point map into $B O_{2}\left(C^{\infty}\right)$, i.e. with a trivial bundle. These are not bordant in $\mathfrak{N}_{4}^{Z_{2}}\left(B O_{2}\left(C^{\infty}\right)\right)$. In $\mathfrak{N}_{5}^{Z_{2}}\left(B O_{2}\left(C^{\infty}\right) \times\left(E Z_{4} / Z_{2}\right)\right)$ they are represented by maps into point $\times E Z_{4} / Z_{2}$, hence come from $\mathfrak{N}_{5}^{Z_{2}}\left(p t \times\left(E Z_{4} / Z_{2}\right)\right)$ $\cong \mathfrak{N}_{5}\left(B Z_{4}\right)$; i.e. one need only consider the bordism classes of the free $Z_{4}$ actions $T_{0} \times i$ and $T_{1} \times i$ on $R P(4) \times S^{1}$.

First dividing out $Z_{2}$ gives $R P(4) \times S^{1}$ with actions $T_{0} \times(-1)$ and $T_{1} \times$ $(-1)$ and dividing $Z_{2}$ again gives the real projective space bundles $R P(5)$ and $R P(2 \lambda \oplus 3)$ over $R P(1), \lambda$ the nontrivial line bundle. Since $2 \lambda$ is trivial, both actions are given by $R P(4) \times S^{1} \stackrel{\pi}{\longrightarrow} S^{1} \hookrightarrow B Z_{4}$ where the map into $B Z_{4}$ classifies the standard cover $z \rightarrow z^{4}: S^{1} \rightarrow S^{1}$. This gives

Proposition 4.1. $\mu \circ \tau_{*}$ is not monic. It does, however, determine the fixed component and fixed data of odd codimension larger than one.

CoROllaRy (Bix). $\chi: \mathfrak{N}_{*}^{G} \rightarrow \operatorname{Hom}\left(H^{*}\left(B O ; Z_{2}\right), H^{*}\left(B G ; Z_{2}\right)\right)$ is not monic if $G=Z_{4}$.

To see that $\mu \circ \tau_{*}$ detects classes not detected by $\chi$ one must explicitly compute some examples. One follows Bix's technique.

It will be convenient to have a method for describing $\operatorname{Hom}\left(H^{*}\left(B O ; Z_{2}\right), A\right)$. Let $f: R P(\infty) \rightarrow B O$ classify the nontrivial line bundle and $b_{i} \in H_{i}\left(B O ; Z_{2}\right)$ the image of the nonzero class in $H_{i}\left(R P(\infty) ; Z_{2}\right)$. Using the ring structure obtained from the Whitney sum, $H_{*}\left(B O ; Z_{2}\right)$ is the $Z_{2}$ polynomial ring with unit $1=b_{0}$ on the $b_{i}$. A homomorphism $\lambda: H^{*}\left(B O ; Z_{2}\right) \rightarrow A$ may then be identified as the class in $H_{*}\left(B O ; Z_{2}\right) \otimes A$ given by $\Sigma b_{i_{1}} \cdots b_{i_{r}} \otimes \lambda\left(s_{\left(i_{1}, \ldots, i_{r}\right)}\right)$ where $s_{\omega}$ form the base in $H^{*}\left(B O ; Z_{2}\right)$ dual to the $b_{\omega}$ in $H_{*}\left(B O ; Z_{2}\right)$.

Let $u, d$ be the nonzero classes in $H^{1}\left(B Z_{4} ; Z_{2}\right)$ and $H^{2}\left(B Z_{4} ; Z_{2}\right)$ so that $H^{*}\left(B Z_{4} ; Z_{2}\right)=Z_{2}[u, d] / u^{2}=0$.

Let $M=R P(2 n+2)$ with the trivial $Z_{4}$ action "id" fixing every point. 
Then $E G \times_{G} M=B G \times R P(2 n+2)$, and $H^{*}\left(E G \times_{G} M ; Z_{2}\right)$ is $H^{*}\left(B G ; Z_{2}\right)[a] / a^{2 n+3}=0$, where $a$ is the nonzero class in $H^{1}\left(R P(2 n+2) ; Z_{2}\right)$. $\alpha^{*}$ is then the homomorphism sending $w$ to $(1+a)^{2 n+3}$ which may be written as $\left(\Sigma_{i} b_{i} a^{i}\right)^{2 n+3}$. Applying integration along the fibers takes the coefficient of $a^{2 n+2}$, so

$$
\chi((R P(2 n+2), \mathrm{id}))=\sum b_{\omega} \otimes s_{\omega}[R P(2 n+2)] \in H_{*}\left(B O ; Z_{2}\right) \otimes H^{\circ}\left(B G ; Z_{2}\right) .
$$

Letting $M=R P(2 n+2)$ with the involution $t\left(\left[x_{0}, x_{1}, \ldots, x_{2 n+2}\right]\right)=$ $\left[-x_{0},-x_{1}, x_{2}, \ldots, x_{2 n+2}\right], E G \times_{G} M$ may be identified with the projective space bundle of the vector bundle $2 \lambda \oplus(2 n+1)$ over $B Z_{4}$, where $\lambda$ is the nontrivial line bundle over $B Z_{4}, w_{1}(\lambda)=u$. Letting $\mu$ be the bundle of vectors in lines in fibers of $2 \lambda \oplus(2 n+1)$, and $a=w_{1}(\mu), H^{*}\left(E G \times_{G} M ; Z_{2}\right)$ is the free module over $H^{*}\left(B G ; Z_{2}\right)$ on $1, a, \ldots, a^{2 n+2}$ with relation $a^{2 n+3}=$ $\Sigma w_{i}(2 \lambda \oplus(2 n+1)) a^{2 n+3-i}$, but $w(2 \lambda \oplus(2 n+1))=(1+u)^{2}=1$, so $a^{2 n+3}=$ 0 . The bundle $E G \times_{G} \tau(M)$ is the tangent bundle $\theta$ to the fibers of $R P(2 \lambda \oplus(2 n+1))$ and $\theta \oplus 1$ is $\mu \otimes \pi^{*}(2 \lambda \oplus(2 n+1))$ so

$$
\begin{aligned}
w(\theta) & =(1+a+u)^{2}(1+a)^{2 n+1}=\left(1+a^{2}+u^{2}\right)(1+a)^{2 n+1} \\
& =\left(1+a^{2}\right)(1+a)^{2 n+1}=(1+a)^{2 n+3} .
\end{aligned}
$$

Thus $\alpha^{*}$ can be written as $\left(\Sigma_{i} b_{i} a^{i}\right)^{2 n+3}$. Applying integration along the fibers takes the coefficient of $a^{2 n+2}$ and thus $\chi((R P(2 n+2), t))=\chi((R P(2 n+2)$, id $))$.

Noting that the fixed set of $t$ is $R P(1) \cup R P(2 n)$ with $R P(2 n)$ not bounding as a manifold, while id fixes $R P(2 n+2)$, these are not bordant involutions. Thus one has

Proposition 4.2.

$$
\chi \circ P: \mathfrak{N}_{*}^{Z_{2}}=\mathfrak{N}_{*}^{Z_{2}}\left(B O_{0}\left(C^{\infty}\right)\right) \rightarrow \operatorname{Hom}\left(H^{*}\left(B O ; Z_{2}\right), H^{*}\left(B Z_{4} ; Z_{2}\right)\right)
$$

is not monic.

Since $P$ is monic, one sees that $\mu^{\circ} \tau_{*}$ distinguishes classes in im $P \subset \mathfrak{V}_{*}^{Z_{4}}$ which are not distinguished by $\chi$.

COROllaRY. ker $\psi$ properly contains $\operatorname{ker} \mu^{\circ} \tau_{*}$ for $G=Z_{4}$.

\section{REFERENCES}

1. M. C. Bix, $Z_{4}$ equivariant characteristic numbers and cobordism (preprint).

2. R. E. Stong, Equivariant bordism and Smith theory, Trans. Amer. Math. Soc. 159 (1971), 417-426. MR 44 \#4779a.

3. Equivariant bordism and Smith theory. II, Trans. Amer. Math. Soc. 162 (1971), 317-326. MR 44 \#4779b.

4. T. tom Dieck, Characteristic numbers of $G$ manifolds. I, Invent. Math. 13 (1971), 213-224. MR 46 \#8236.

DEPARTMENT OF MATHEMATICS, UNIVERSITY OF VIRGINIA, CHARLOTTESVILLE, VIRGINIA 22903 